\title{
Calyceal diverticular stones: Does the insistence on less invasive technique keep the patient away from a major complication?
}

\author{
Murat Tugrul EREN ${ }^{1}$ (D), Hakan OZVERI ${ }^{2}$ (D) \\ ${ }^{1}$ Operating Room Services, Vocational School of Health Sciences, Acibadem Mehmet Ali Aydınlar University and Urology Clinic, Acibadem Kozyatagi \\ Hospital, Istanbul, Turkey. \\ ${ }^{2}$ Department of Urology, School of Medicine, Acibadem Mehmet Ali Aydinlar University, Istanbul, Turkey.
}

Corresponding Author: Murat Tugrul EREN

E-mail: murat.eren@acibadem.com

Submitted: 17.01.2021 Accepted: 28.04.2021

\begin{abstract}
Calyceal diverticular stones are rare clinical entities without a clearly defined consensus on the treatment. Treatment indications include recurrent urinary tract infections, chronic pain and renal impairment. Treatment modality of surgical approach is usually based on the location of calyceal diverticulum.

A 23-year-old woman was presented with a calyceal diverticular stone of $16.5 \mathrm{~mm}$. Her medical history revealed multiple urinary tract infections caused by extended spectrum beta-lactamase (ESBL)-producing Escherichia coli of which the stone was predicted to be the reason. The patient was treated ureterorenoscopically, a decision of which was taken intra-operatively.

In conclusion, insisting on performing nephroscopy following an intra-operative retrograde pyelography revealed no apparent ostium which could be interpreted as ureterorenoscopic retriaval would fail and development of postoperative bacteremia despite all precautions taken pre-and intra-operatively were two lessons indicating that all efforts must be exercised to reach the most minimal invasive method for the treatment of calyceal diverticular stones.

Keywords: Calyceal kidney stone, Endoscopic treatment, Flexible ureteroscopy
\end{abstract}

\section{INTRODUCTION}

Calyceal diverticular stones are rare clinical entities without a clearly defined consensus on treatment [1]. Treatment indications include recurrent urinary tract infections, chronic pain, and renal impairment [2]. The location of the diverticulum in the kidney usually determines the treatment modality or surgical approach for stones, and infundibular length and opening of the diverticulum may affect not only the treatment modality, but also the success rates [3]. Ureterorenoscopic retrieval of the stone in a calyceal diverticulum is recommended for small-sized stones located in the upper/mid and anterior locations, whereas, percutaneous nephrolithotomy (PCNL) is recommended for larger stones and lower and posterior locations, yielding higher stone-free rates [4]. However, infectious and major complications rates are still higher with PCNL.

Herein, we report a 23 -year-old patient presenting with a calyceal diverticular stone treated by ureterorenoscopic stone retrieval technique.

\section{CASE REPORT}

A 23-year-old female patient with a body mass index of $20,7 \mathrm{~kg} /$ $\mathrm{m}^{2}$ was referred to our urology outpatient clinic due to recurrent urinary tract infections for the past two years. During her physical examination, left costovertebral angle sensitivity was detected. An ultrasonographic evaluation revealed an intraparenchymal, hyperechogenic lesion in the upper posterior pole, measured maximally at $16.5 \mathrm{~mm}$ in size. The hyperechogenic lesion was suspicious for intra-parenchymally located kidney stone trapped behind the narrowed infundibula of the diverticulum. The parenchyma over the stone was very thin. A non-contrast computed tomography (CT) was performed for further evaluation, as the patient refused intravenous (IV) contrast media injection. A very small infundibular opening of the diverticulum was barely predicted at the CT cross-sections (Fig. 1). Decision of surgical intervention for the stone was taken and a written informed consent was obtained from the patient for both antegrade and retrograde approach.

The patient was given $1 \mathrm{~g}$ of meropenem (IV) preoperatively as prophylactic antibiotherapy, as the patient had a history of multiple urinary tract infections caused by resistant bacteria, although preoperative culture was sterile. Under general anesthesia, retrograde pyelogram (RPG) was performed using an $8 \mathrm{~F}$ open-end ureteral catheter inserted to the left collecting system. Particular care was exercised not to increase the

How to cite this article: Eren MT, Ozveri H. Calyceal diverticular stones: Does the insistence on less invasive technique keep the patient away from a major complication? Marmara Med J 2021; 34: 344-347, doi: 10.5472/marumj.1013465 
intrapelvic pressure, as the patient was still carrying a risk for bacteremia. Despite waiting for 3 to $4 \mathrm{~min}$, the diverticulum was seen not to be filled with contrast media, which could be an indicator of stenotic infundibulum under fluoroscopy (Fig. 2). Subsequently, diagnostic flexible ureterorenoscopy (fURS) was performed to confirm the anatomy. An 8-10F ureteral access sheath of $35 \mathrm{~cm}$ in length was inserted into the upper ureter under fluoroscopic guidance over the guidewire. A millimetric punctuate ridge, which was thought to be the possible ostium of the diverticulum, was able to be visualized at the upper posterior pole during fURS (Fig. 3). A 0.035-inch guidewire was advanced through this ridge, entering the diverticulum and passing as confirmed by fluoroscopy. Using holmium laser, a precise cut of the mucosa beneath the guidewire and close to the infundibular ostium was done, allowing the entrance into the diverticulum. Lithotripsy was performed with a 270-micron holmium laser probe (Ho: Yttrium Aluminum Garnet (YAG) Laser; Dornier MedTech GmbH, Munich, Germany), after the stone was monitored with flexible ureterorenoscopy (Flex-X2, Karl Storz SE \& Co. KG, Tuttlingen, Germany). Stone basketing was performed by tipless nitinol baskets (Zero Tip ${ }^{\mathrm{TM}}$; Dakotta ${ }^{\mathrm{TM}}$ Boston Scientific Corp., MN, USA). During the operation, the following settings were used for the laser energy: 10-20 Hz frequency and a power of 0.4-1.0 Joule. Dusting, popcorn and fragmentation modes were used for stone management. No residual stone fragment was left. Nephroscopic evaluation was also performed to confirm that no stones were migrated from the diverticulum to the other calyces and, finally, RPG revealed an intact infundibulum of the diverticulum (Fig. 4). The procedure was ended by inserting a 4.8F, $26 \mathrm{~cm}$ DJ stent with a magnetic tip (Black-Star; Urotech GmbH, Achenmühle, Germany) and catheterization of the bladder with a 16F Foley catheter.

At the fifth postoperative hour, the patient developed serious hypotension $(70 / 40 \mathrm{mmHg})$, high fever $\left(38.5^{\circ} \mathrm{C}\right)$, and shivering. Complete blood count and laboratory analysis revealed a white blood cell count of $19,000 / \mathrm{mm}^{3}$ and elevated C-reactive protein (CRP) values. The platelet count also decreased with abnormal liver function tests. After taking blood culture samples, continuous high-flow $(250 \mathrm{ml} / \mathrm{h})$ IV fluid was given to achieve an optimal blood pressure. As the patient was clinically accepted as systemic inflammatory response syndrome (SIRS) with infection, IV antibiotic regimen was continued with meropenem ( $1 \mathrm{~g}$ t.i.d.). With supportive treatment, the patient recovered gradually without any sequelae. At postoperative day 5 , all biochemical and clinical parameters of SIRS resolved, and the patient was discharged with oral antibiotherapy for 21 days.

On postoperative day 15, ultrasonographic evaluation showed a normal left kidney without a stone inside and the DJ stent was removed. Stone analysis revealed a mixed stone type containing calcium oxalate and carbonate apatite. Diet and proper recommendations according to the stone type was given to the patient. At three months of follow-up, there was no stone in the left kidney and a very small-sized diverticulum was visualized on ultrasonography. The patient is still free from symptoms and urinary tract infection at eight months of follow-up.

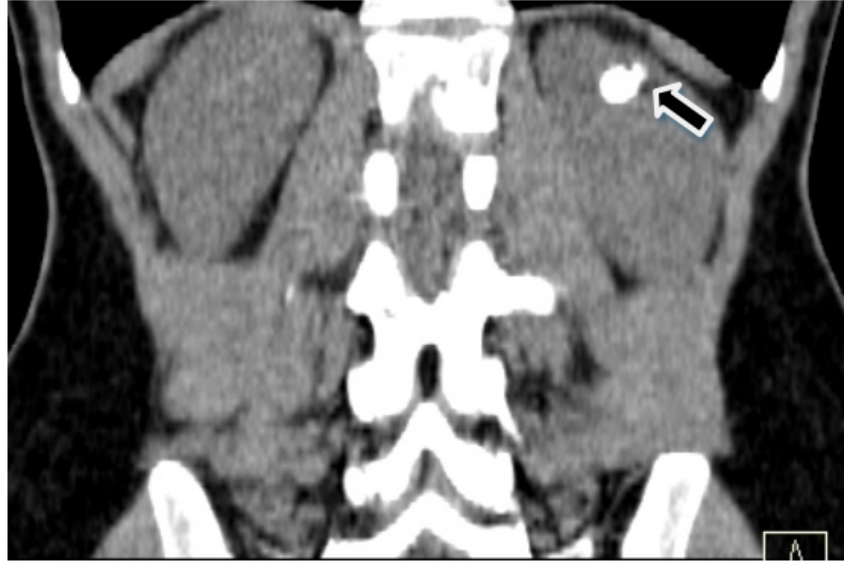

Figure 1. A non-contrast computed tomography image showing very small infundibular opening of the diverticulum.

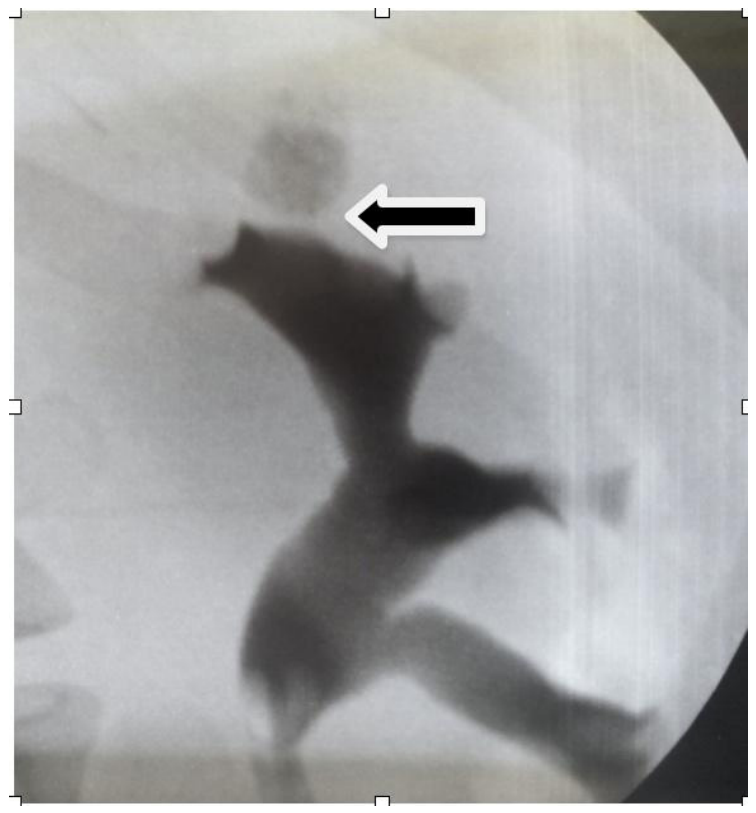

Figure 2. Diverticulum not filled with contrast agent.

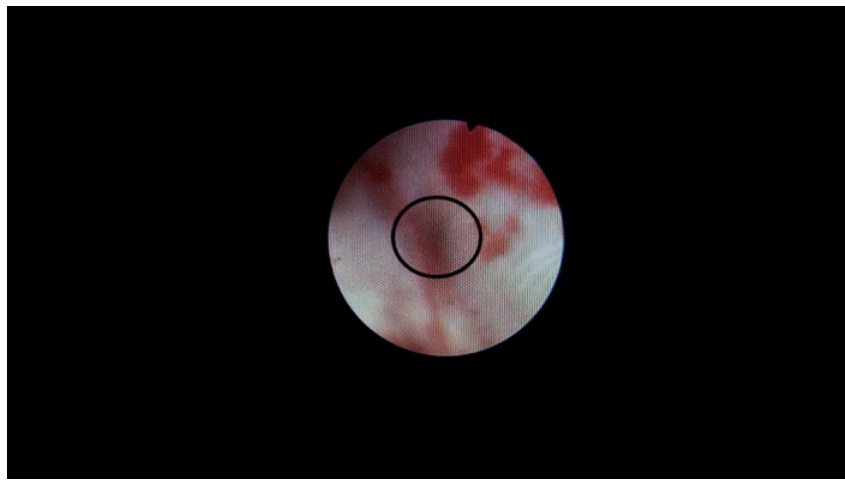

Figure 3. A view of ostium-like ridge at upper pole posteriorly during flexible ureterorenoscopy. 


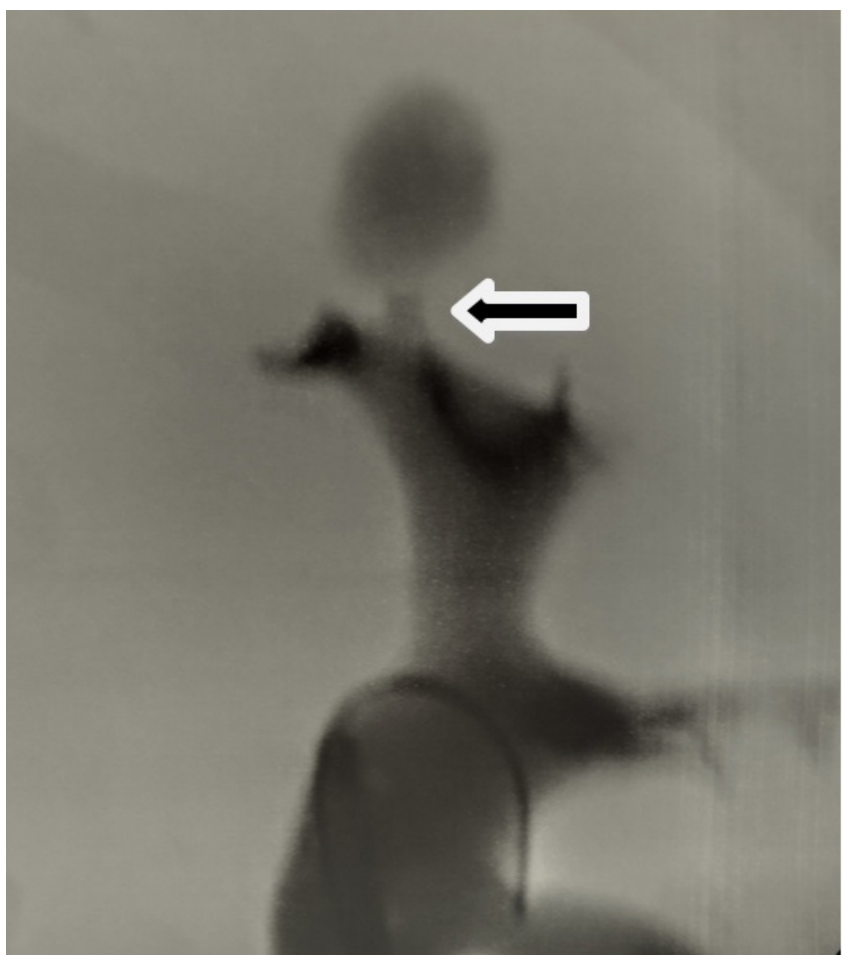

Figure 4. Retrograde pyelography showing an intact infundibulum of the diverticulum after removal of stones.

\section{DISCUSSION}

Calyceal diverticula are very rare abnormalities with an incidence of less than $1 \%$ [1]. They communicate with the collecting system through an infundibulum [2], and the diameter and length of the infundibulum are the main determinants of complications. The most common indication for surgical treatment of a calyceal diverticulum is the presence of calculus, which accounts for 9.5 to $39 \%$ of cases [2]. The presented case had a stone in the diverticulum, but indication for surgery was recurrent urinary tract infections with resistant bacteria, which is one of the indications for the treatment [3].

Although, preoperative non-contrast CT imaging may have resulted in inadequate evaluation of the diverticular infundibulum, we believe that this did not affect our treatment strategy at all for two main reasons. First, as in case of a narrow or stenotic infundibulum, contrast agent cannot fill these nonsecretory cavities and, therefore, retrograde filming is still needed. Second, we planned to decide the treatment modality intraoperatively via RPG in our case. The reason for this was the preference of ureterorenoscopic intervention rather than percutaneous approach due to possible infectious complications and the fact that the patient had a history of multiple urinary tract infections with resistant bacteria. Thus, we recommend ureterorenoscopic treatment to avoid infectious complications in eligible cases.
In general, PCNL is preferred for such stones to achieve a stone-free status, particularly for a large diverticulum with a large stone burden [4]. Although, PCNL is more invasive than ureteroscopy (URS), it has the highest stone - and symptomfree rates ( $>90 \%)$ [5]. The reported incidence rates of infectious complications following PCNL are as follows: bacteremia $23 \%$; endotoxemia $34 \%$; fever $25 \%$; and septic shock 0.3 to $2.5 \%$ [4]. On the other hand, postoperative infectious complications of ureterorenoscopy were reported in $2.97 \%$ of cases, of which $0.3 \%$ developed severe sepsis, which is slightly lower than PCNL with the latter, also having a higher rate of major complications up to $5.5 \%$ (i.e., hemo-pneumothorax, pneumothorax, and hemorrhage).

In the presented case, RPG was not successful to reveal the infundibular neck intraoperatively (Fig. 3). Therefore, PCNL decision could be made without a nephroscopic evaluation, as the major drawback of fURS is locating the diverticulum, since its neck may be narrow and even concealed [6]. In such a case, the fURS procedure must be converted to PCNL without any need for nephroscopy. However, insiting on fURS treatment was our drive to do nephroscopy in our case. Therefore, we recommend performing nephroscopy to check the diverticular orifice, even the RGP did not reveal it. During ureterorenoscopic stone retrieval, using the ureteral access sheath and keeping the irrigation pressure low, we attempted not to increase the intrarenal pressure, which may cause pyelovenous backflow and absorption, which are both proven risk factors for infectious complications. The holmium laser was manipulated very gently to avoid bleeding for the same reason. In addition, as sterile preoperative urine culture does not correspond with the infection present in the upper urinary tract, we used a potent, broad-spectrum antibiotic for preoperative prophylaxis. However, despite all these intraoperative efforts, including the empiric use of a broad-spectrum antibiotic, our case developed clinical signs and symptoms of postoperative urosepsis and was diagnosed with urosepsis based on the Sequential Organ Failure Assessment (SOFA) score. We believe that the reason for the negative urine and blood cultures obtained during this period may be due to the use of prophylactic antibiotherapy, namely meropenem.

Nonetheless, complete stone-free and infection-free status of our patient confirmed that the cause of the resistant urinary tract infections was the calyceal diverticular stones, although, infections were treated with the most appropriate antibiotic regimen. In a percutaneous approach, complications would be expected to be higher in this complicated case.

\section{Conclusion}

In conclusion, treatment of calyceal diverticular stones can be challenging in some cases. Minimally invasive management of this clinical scenario with retrograde intrarenal surgery is more effective than other treatment modalities in selected cases. Treatment must be tailored according to the anatomical features of each individual patient and experience of the surgical team. This case highlights the utility of less invasive techniques with careful handling to avoid more serious complications, keeping 
in mind that serious complications still may occur, despite all precautions and maneuvers.

Conflict of interest: The authors have no conflicts of interest to declare.

Funding: No financial support was received for this study.

Authors' contributions: M.T.E.: Conception and design of the study. Performance of ureterorenoscopic operation. Collection, analysis and interpretation of the data. Drafting the article and revising it critically for important intellectual content. H.O.: Design of the study. Performance of the ureterorenoscropic operation. Collection and interpretation of the data. Drafting the article and revising it critically for important intellectual content. Both authors approved the final version of the article.

\section{REFERENCES}

[1] Turna B, Raza A, Moussa S, Smith G, Tolley DA. Management of calyceal diverticular stones with extracorporeal shock wave lithotripsy and percutaneous nephrolithotomy: long-term outcome. BJU Int 2007;100:151-6. doi:10.1111/j.1464-410X. 2007.06911.x

[2] Timmons JW Jr, Malek RS, Hattery RR, Deweerd JH. Caliceal diverticulum. J Urol 1975;114: 6-9. doi: https://doi. org/10.1007/978-1-59259-954-7_14

[3] Rapp DE, Gerber GS. Management of caliceal diverticula. J Endourol 2004;18:805-10. doi: 10.1089/end.2004.18.805.

[4] Waingankar N, Hayek S, Smith AD, et al. Calyceal diverticula: A comprehensive review. Rev Urol 2014; 16: 29-43.

[5] Patodia M, Sinha RJ, Singh S, Singh V. Management of renal caliceal diverticular stones: A decade of experience. Urol Ann 2017;9:145-9. doi: 10.4103/UA.UA_95_16.

[6] Zhang JQ, Wang Y, Zhang JH, Zhang XD, Xing NZ. Retrospective analysis of ultrasound-guided flexible ureteroscopy in the management of calyceal diverticular calculi. Chin Med J (Engl) 2016;129:2067-73. doi: 10.4103/03666999.189060 . 